\title{
A Review of Binocular Treatment for Amblyopia
}

\author{
Lampros Lamprogiannis, ${ }^{1,2}$ Daisy MacKeith ${ }^{3}$ and Anthony J Vivian ${ }^{3}$ \\ 1. King's College Hospital NHS Trust, London, UK; 2. Great Ormond Street Hospital, London, UK; \\ 3. Addenbrooke's Hospital NHS Trust, Cambridge UK
}

DOI: https://doi.org/10.17925/EOR.2020.14.1.34

A mblyopia has traditionally been regarded as a monocular condition and its treatment (refractive correction and penalisation) has been aimed at the improvement of vision in the amblyopic eye. However, current approaches emphasise the importance of binocular function of unilateral amblyopes and point to binocular treatment as a potentially valuable way to achieve therapeutic results. Perceptual learning with both eyes open and dichoptic training, where a different image is presented to each eye, have demonstrated significant results, aiming to address interocular suppression as the primary cause of strabismic and refractive amblyopia. Advancements in digital technology have allowed the application of a number of devices (laptop, iPod, iPad, Oculus Rift), with appropriate software, to the binocular dichoptic treatment of amblyopia. Further research and well-prepared studies of adequate size may provide a more definite answer as to the value of binocular treatment and its role in the management of amblyopia.

\section{Keywords}

Amblyopia, perceptual learning, dichoptic training, binocular treatment

Disclosures: Lampros Lamprogiannis, Daisy MacKeith and Anthony J Vivian have no financial or non-financial relationships or activities to declare in relation to this article.

Review Process: Double-blind peer review.

Compliance with Ethics: This study involves a review of the literature and did not involve any studies with human or animal subjects performed by any of the authors.

Authorship: The named authors meet the International Committee of Medical Journal Editors (ICMJE) criteria for authorship of this manuscript, take responsibility for the integrity of the work as a whole, and have given final approval for the version to be published.

Access: This article is freely accessible at

tOuChOPHTHALMOLOGY.COM

(c) Touch Medical Media 2020.

Received: 28 November 2019

Accepted: 28 February 2020

Published Online: 29 April 2020

Citation: European Ophthalmic Review. 2020;14(1):34-8

Corresponding Author: Anthony J Vivian,

Department of Ophthalmology, Box 41,

Addenbrooke's Hospital, Cambridge, CB2 OQQ, UK

E: vivianeye@aol.com

Support: No funding was received for

the publication of this article.
The term amblyopia, derived from the Greek words $\alpha \mu \beta \lambda u^{\prime}$ (weak) and ${ }^{\prime \prime} \psi($ (eye), is defined as a unilateral (or, less commonly, bilateral) reduction of visual acuity as a result of inadequate visual experience during early life, which leads to a disruption of the normal visual neurodevelopment process. ${ }^{1}$ It is associated with anisometropia, ametropia, stimulus deprivation, strabismus and nystagmus, and it is considered one of the most common causes of unilateral visual impairment that presents in childhood and persists into adult life. ${ }^{2}$ Its prevalence is approximately $3 \%$ of the UK population and it affects both the individual and community in terms of screening and treatment. ${ }^{3}$ Traditionally, amblyopia has been regarded as a monocular disease and, therefore, the mainstay of its treatment has been the penalisation of the fellow normal eye using patching or atropinisation, aiming to increase the visual stimulation of the amblyopic eye and to eliminate its suppression.

The cause of the amblyopia needs to be addressed, either prior to amblyopia therapy or simultaneously (refractive correction, correction of occlusive causes such as cataract and ptosis, management of strabismus). Perceptual learning, which relies on the use of the amblyopic eye while participating in engaging activities, has proved to be a valuable added tool to the traditional therapeutic methods. However, it has now been established that, despite their reduced binocular visual functions, such as stereoacuity, many amblyopes maintain the capacity for binocular interaction; this finding has led researchers to explore the efficacy of treatment of amblyopia through binocular stimulation. ${ }^{4}$

Binocular therapies for amblyopia include perceptual learning, which was defined by Eleanor Gibson in 1963 as 'any relatively permanent and consistent change in the perception of a stimulus array, following practice or experience with this array', ${ }^{5}$ and dichoptic training. ${ }^{6}$ In this review, we aim to present the binocular therapies for unilateral amblyopia and to examine their effectiveness. A literature review was performed by using the search term 'binocular treatment of amblyopia' in the National Center for Biotechnology Information database, and research published since 2012, a period which coincides with an increased interest in binocular amblyopia treatment methods, was included in the present review. Nineteen studies have been included in this review, 15 of which support the value of binocular treatment of unilateral amblyopia (78.9\%) (Table 1).

\section{New perceptions in the pathophysiology of amblyopia}

Monocular amblyopia demonstrates a higher prevalence than binocular amblyopia. Disruption of the visual maturation process by amblyogenic factors has been considered to lead to structural and functional changes to the visual cortex and it has been widely accepted that these changes are irreversible after childhood. These beliefs have defined the diagnostic and therapeutic approach to amblyopia until recently. However, current treatments demonstrate poor results in a large proportion of children treated with penalisation of the healthy eye, and is essentially ineffective in adolescents and adult patients. ${ }^{6.7}$ These limitations have led to further research into the mechanism of amblyopia and its treatment options. 


$$
\mid
$$


It is now a common belief that uniocular amblyopes retain intact binocular processes that, opposed to traditional understanding, have not been irretrievably lost, but are suppressed; therefore, amblyopes are considered to retain the capacity of binocular processes if suppression is appropriately addressed. ${ }^{8}$ Adults' visual systems have been found to demonstrate plasticity and may, therefore, benefit from treatment aiming to decrease suppression and to restore binocular functions. ${ }^{9}$ This change in our perceptions has caused an increased interest in the binocular treatment of amblyopia, as amblyopes' monocularity is now regarded as functional and not as structural. Perceptual learning and dichoptic training are the principle focus of current research, with some early evidence of positive results.

\section{Perceptual learning}

The use of perceptual learning techniques has been the focus of many studies predominately investigating the reversal of established amblyopia in adults. Modern technology advancements in the field of informatics have enabled researchers to use appropriate interactive software to improve visual performance. Binocular perceptual learning strategies assume that amblyopia is a result of interocular inhibition, which is a natural mechanism of the body to remove binocular diplopia. Some studies of perceptual learning in the treatment of amblyopia have encountered strong criticism, which has mostly focused on the small sample of related studies, the limitations of home-based training and the lack of long-term follow-up. ${ }^{10,11}$

Recent studies have demonstrated increased contrast sensitivity, improvement in visual acuity and letter-recognition tasks, as well as a reduction of the crowding effect via repetition of visual tasks. ${ }^{10}$ Contrary to the criticism that these gains are limited to these specific tasks, Levi provides evidence that participants' improvement is actually durable and generalised. ${ }^{11}$ It is noteworthy that monocular perceptual training has been shown to lead to an improvement in binocular vision. ${ }^{12,13}$ Ooi et al. have described a binocular 'push-pull' protocol based on the principles of perceptual learning, which simultaneously reduces sensory eye dominance and excites the weak eye. ${ }^{14}$ This protocol has produced promising results, as targeting the inhibition mechanism of interocular rivalry has significantly reduced suppression and improved stereopsis; at the same time, it has provided further evidence in favour of the binocular nature of amblyopia.

\section{Interactive Binocular Treatment project}

The Interactive Binocular Treatment (I-BiT) project was set up to treat amblyopia with video games/video footage viewed with 3D viewing technology. ${ }^{15}$ The principle being that amblyopia was a binocular problem requiring a binocular solution which dichoptic training is able to deliver. The I-BiT system allowed both eyes to receive an image but crucial parts of the image were seen only by the amblyopic eye. Treatment involved either playing computer games in which objects of interest (such as coins to collect) went only to the amblyopic eye or watching short films with footage sent only the amblyopic eye within a border/background common to both eyes.

Results from an early I-BiT prototype were promising; children who had no success with patching treatment demonstrated improvements in visual acuity with I-BiT (mean 0.250 LogMAR; $n=6$ ) with a mean total treatment duration of just 4.4 hours and treatment was enjoyable for children. ${ }^{15}$ Advances in 3D viewing technology from the gaming industry allowed for a more user-friendly/mobile I-BiT system using shutter glasses. Shutter glasses turn off and on rapidly, alternating between right and left lens in sync with the 'refresh' rate of a computer monitor so that a different image can be sent to each eye. A pilot study found a clinically significant improvement in visual acuity (mean 0.18 LogMAR: $\mathrm{n}=9$ ) with a treatment duration of only 30 minutes per week (current patching regimens for amblyopia are 2-6 hours per day). ${ }^{16}$ However, Herbison et al. found much smaller gains in visual acuity (mean 0.07 LogMAR) with no difference between treatment arms in a randomised, controlled, double-masked trial of 75 children aged 4-8 years. ${ }^{17}$ The control group in this trial also used the shutter glasses to play a computer game, but the same image was sent to both eyes. The majority of the participants in the trial had received previous patching treatment (76\%) and therefore their potential for visual acuity improvement was limited, but the results were equally disappointing for the few amblyopes who had received no previous patching treatment. Further limitations of this study included a short period of therapeutic intervention per week, a short total treatment duration (3 hours) and the inclusion of strabismic amblyopes without a mechanism to compensate for angle of deviation. A new trial is underway of a home-based I-BiT system which can deliver a daily treatment dose and which can offset the angle of deviation in strabismic eyes to allow bifoveal fixation. The trial is recruiting treatment-naive anisometropic, strabismic and mixed amblyopes (ClinicalTrials.gov identifier: NCT02810847). ${ }^{18}$

\section{iPod binocular home-based treatment for amblyopia}

In a study by Hess et al., efficacy and compliance of dichoptic training were evaluated both at home and in the clinic.19,20 This method is based on previous findings that underline the crucial role of binocular functions and suggest that these actually remain intact even in adult amblyopes; ${ }^{21}$ it is the interocular suppression that renders their visual system functionally monocular. As it was established that dichoptic training produced promising results, researchers proceeded to incorporate this method in a handheld iPod device, using the popular video game Tetris. Different elements of the game were displayed to the two eyes of adult amblyopes, initially in a clinic setting, and a combination of the two images was necessary to successfully play the game. Suppression was gradually reduced in the majority of the participants, and subsequent improvement of visual acuity and stereopsis was noted. Home-based studies led to equally good outcomes and researchers have concluded that this approach may combine convenience with satisfactory outcomes. This is important as adult amblyopes have long been considered to be unresponsive to conventional treatment of amblyopia. The small size of the study sample, as well as the limited duration of the follow-up period, constitute obvious limitations to the value of this otherwise well planned and executed study.

\section{Binocular iPad treatment for amblyopia}

The value of dichoptic iPad game play in the treatment of amblyopia has been thoroughly examined in three significant studies at the Retina Foundation of the Southwest in Texas, USA. Li et al. assessed the value of binocular iPad game play in children between the ages of 4 and 12 years with stable visual acuity upon initiation of the study.2 Rapid improvement of visual acuity was noted; however, compliance was not optimal. Kelly et al. utilised a more sophisticated computer game (when compared to relatively simple Tetris and Pong) and examined the value of this binocular treatment in children between 4 and 10 years of age. ${ }^{23}$ This led to better compliance, gain in visual acuity and reduction of suppression. Children between the age of 3 and 7 years also seemed to benefit from dichoptic treatment with iPad games, as Birch et al. demonstrated. ${ }^{24}$

It is worth mentioning that the Paediatric Eye Disease Investigator Group designed and conducted a multicentre randomised clinical trial to 
assess the value of dichoptic training of amblyopia with the use of iPad games in 2019. ${ }^{25}$ This trial did not demonstrate an improvement of visual acuity and stereoacuity for children who underwent binocular treatment compared with optical treatment alone, despite good compliance with the treatment plan. Investigators have underlined that this trial did not include children of pre-school age who might have responded better. At the same time, questions were raised regarding the lack of dose-response relationship of binocular treatment and, as a result, regarding its exact physiological interpretation.

\section{Binocular Treatment of Amblyopia Using Videogames clinical trial}

The Binocular Treatment of Amblyopia Using Videogames (BRAVO) study team developed a protocol to assess the efficacy of dichoptic training with the use of video games in a home-based setting over a period of 6 weeks in children greater than 7 years old and in adults. ${ }^{26}$ This multicentre, double-masked, randomised clinical trial took place between March 2014 and June 2016 and the primary outcomes that were measured were visual acuity in the amblyopic eye, compliance, stereoacuity and quality of life.

This trial was based on the belief that interocular suppression is the predominant pathogenetic mechanism in amblyopia. However, results after 6 weeks of treatment did not meet the researchers' expectations, as the group that underwent the treatment did not achieve better outcomes than the placebo group. ${ }^{27}$ Poor compliance, dose-continuity effects or a failure of the particular method have been suggested possible causes of these results. Researchers underline the need for further testing of dichoptic training prior to reaching a definite conclusion regarding its value and its place in the treatment of amblyopia.

\section{Dichoptic training using head-mounted displays}

Dichoptic training is a visual training process involving the presentation of different stimuli to each eye; in order to achieve the tasks, the summation of both stimuli is required. For instance, the object of the game may be presented to the amblyopic eye and the background to the other eye. This contrasts to perceptual learning tasks in which a single identical stimulus is presented to each eye. Dichoptic training relies on improving binocular visual function and thus addresses the suppression of the amblyopic eye, leading to a gradual overall improvement in visual function in terms of visual acuity and sensory function.

Žiak et al. conducted an interesting study, based on the principles of simultaneous binocular dichoptic training for adult amblyopes. ${ }^{28}$ The novel, virtual reality, head-mounted device Oculus Rift (Oculus, Menlo Park, CA, USA) was utilised and participants were invited to play two appropriate computer games with a dichoptic setting, twice a week, over a period of 4 weeks in total. The majority of the participants demonstrated significant improvement in visual acuity of the amblyopic eye, as well as in stereopsis. This is in agreement with previous dichoptic training studies and confirms the hypothesis of residual cortical plasticity in the adult brain. This study demonstrates important limitations such as a small sample size, a short follow-up and an absence of a control group; it should also be noted that adult amblyopes present the added challenge of a variable previous history of treatment for amblyopia, which renders comparison difficult.

Knox et al. have also applied a head-mounted device that allows prolonged dichoptic stimulation. ${ }^{29}$ Participants were asked to play a simple game that required binocular interaction. It is noteworthy that children with small angle or no squint benefited the most from this treatment in terms of visual acuity and stereo-function and the authors reasonably underline the need for distinction between different types of amblyopia.

\section{Balanced binocular viewing therapy}

Bossi et al. have described a new approach to binocular treatment of amblyopia, which they have named 'balanced binocular viewing therapy'. ${ }^{30}$ This relies on watching 3D, dichoptic movies while wearing specially designed goggles, combined with an interactive game that aims to measure suppression. This technique has demonstrated promising results (significant improvement in visual acuity of the amblyopic eye and increased stereoacuity in patients with some baseline stereoacuity). While the treatment period varied between 8 and 24 weeks, compliance was overall deemed satisfactory. Interestingly, the simultaneous measurement of interocular suppression does not support its significance as a cause of amblyopia, according to the authors.

\section{Discussion}

Optimal management of amblyopia remains at the epicentre of paediatric ophthalmology research, as amblyopia accounts for a significant part of the workload of paediatric ophthalmologists worldwide, and conventional treatment approaches do not always lead to improvement of vision. Suboptimal compliance is certainly one of the main limitations of the current treatment modalities. Binocular treatment has attracted significant attention from a number of research teams who have attempted to combine experimental findings and technology advances into novel therapeutic models. This is an ongoing process and an objective evaluation of the current research may highlight their findings and, at the same time, underline the challenges that still need to be met.

A critical assessment of the current literature reveals that multiple research groups have designed and conducted interesting studies with promising but limited outcomes. Relying on the contemporary approach of amblyopia as a binocular, rather than a monocular phenomenon, they have managed to implement modern technology in amblyopia treatment. These studies have largely been conducted at home, which is a significant advantage, as they simulate real-world conditions better than clinic-based studies. Some improvement in visual acuity and stereoacuity was noted in all studies under examination; these findings validate the scientific hypothesis of the binocular dysfunction as the main amblyogenesis mechanism.

At the same time, it is important to stress that all of the aforementioned studies have demonstrated significant limitations. Age of participants varied between the studies and this hinders the extraction of solid conclusions, given the importance of age in visual development. Similarly, previous treatment may have affected the outcomes of the studies, ${ }^{17}$ as participants' groups were not always consistent as to this parameter. Finally, compliance with the treatment protocol is a valid concern, despite researchers' efforts to increase it, as poor adherence is a known risk with home-based studies.

These study limitations may explain some of the rather disappointing outcomes of the randomised control trials that have been presented. ${ }^{17,25,27}$ Indeed, the value of binocular treatment for amblyopia has not yet been confirmed by a randomised controlled trial. There is reasonable argument that a different study design might prove more valuable in distinguishing between different subgroups of amblyopes and proving whether some of these subgroups (patients without previous treatment, younger patients, anisometropic amblyopes) may benefit more from binocular treatment. Adjusting treatment dosages to particular age groups of patients and 
carefully addressing adherence to treatment protocols are features of some current controlled trials and may provide more definitive evidence of a significant treatment effect of binocular amblyopia therapies..$^{18}$ At the same time, continuous advancement of technology will provide more sophisticated tools that may well support future research, along with further research regarding the mechanisms of amblyogenesis. With future modifications in delivery, binocular treatment of amblyopia may prove to be a valuable therapeutic modality.
1. Birch EE. Amblyopia and binocular vision. Prog Retin Eye Res. 2013:33:67-4

Tailor V, Bossi M, Bunce C, et al. Binocular versus standard occlusion or blurring treatment for unilateral amblyopia in children aged three to 2015:CD011347.

3. Webber AL. The functional impact of amblyopia

Clin Exp Optom. 2018;101:443-50

4. Hess RF, Thompson B, Baker DH. Binocular vision in amblyopia: structure, suppression and plasticity. Ophthalmic Physiol Opt. 2014;34:146-62

5. Gibson EJ. Perceptual learning. Annu Rev Psychol. 1963;14:29-56.

6. Kraus $\mathrm{CL}$, Culican $\mathrm{SM}$. New advances in amblyopia therapy I: binocular therapies and pharmacologic augmentation. Br J Ophthalmol. 2018;102:1492-6.

7. Wong AM. New concepts concerning the neural mechanisms of amblyopia and their clinical implications. Can I Ophthalmol. 2012:47:399-409.

8. Dahlmann-Noor A. Binocular treatment of amblyopia in children: teething problems on the path to clinical practice. IAMA Ophthalmol 2016:134:1400-1.

9. Shuai L, Leilei Z, Wen W, et al. Binocular treatment in adult amblyopia is based on parvocellular or magnocellular pathway. Eur $J$ Ophthalmol. 2020:30:658-67.

10. Papageorgiou E, Asproudis I, Maconachie G, et al. The treatment of amblyopia: current practice and emerging trends. Graefes Arch Clin Exp Ophthalmol. 2019;257:1061-78

11. Levi DM. Prentice award lecture 2011: removing the brakes on plasticity in the amblyopic brain. Optom Vis SCi. 2012;89:827-38.
12. Chen Z, Li J, Liu J, et al. Monocular perceptual learning of contrast detection facilitates binocular combination in adults with anisometropic amblyopia. Sci Rep. 2016;6:20187.

13. Zhang JY, Cong $\sqcup$, Klein SA, et al. Perceptual learning improves adult amblyopic vision through rule-based cognitive compensation. Invest Ophthalmol Vis SCi. 2014:55:2020-30.

14. Ooi TL, Su YR, Natale DM, He ZJ. A push-pull treatment for strengthening the 'lazy eye' in amblyopia. Curr Bid. 2013;23:R309-10.

15. Waddingham PE, Butler TK, Cobb SV, et al. Preliminary results from the use of the novel Interactive binocular treatmen (I-BiT) system, in the treatment of strabismic and anisometropic amblyopia. Eye (Lond). 2006;20:375-8.

16. Herbison N, Cobb S, Gregson R, et al. Interactive binocular treatment (I- BiT) for amblyopia: results of a pilot study of 3D shutter glasses system. Eye (Lond). 2013;27:1077-83.

17. Herbison N, MacKeith D, Vivian A, et al. Randomised controlled trial of video clips and interactive games to improve vision in children with amblyopia using the I-BiT system. Br J Ophthalmol. 2016:100:1511-6.

18. Brown $R$, Blanchfield $P$. Fakis A et al Clinical investigation plan for the use of interactive binocular treatment (BBiT) for the management of anisometropic, strabismic and mixed amblyopia in children aged 3.5-12 years: a randomised, controlled clinical trial. Trials. 2019:20:427.

19. Hess RF, Thompson B, Black JM, et al. An iPod treatment of amblyopia: an updated binocular approach. Optometry. 2012;83:87-94

20. Hess RF, Babu RJ, Clavagnier S, et al. iPod binocular treatment for amblyopia in adults. Clin Exp Optom. 2014;97:389-98.

21. Hess RF, Mansouri B, Thompson B. A new binocular approach to the treatment of amblyopia in adults well beyond the critical period of visual development. Restor Neurol Neurosci. 2010;28:793-802

rale SE, et al. A binocular iPad treatment for amblyopic children. Eye (Lond). 2014:28:1246-53.

23. Kelly KR, Jost RM, Dao L, et al. Binocular iPad game vs patching for treatment of amblyopia in children: a randomized clinical trial. JAMA Ophthalmol 2016:134:1402-8.

24. Birch EE, Li SL, Jost RM, et al. Binocular iPad treatment for amblyopia in preschool children. J AAPOS. 2015;19:6-11.

25. Holmes JM, Manny RE, Lazar EL, et al. A randomized trial of binocular dig rush game treatment for amblyopia in children aged 7 to 12 years. Ophthalmology. 2019;126:456-66.

26. Guo CX, Babu RJ, Black JM, et al. Binocular treatment of amblyopia using videogames (BRAVO): study protocol for a randomized controlled trial. Trials. 2016;17:504

27. Gao TY, Guo CX, Babu RJ, et al. Effectiveness of a binocular video game vs placebo video game for improving visua functions in older children, teenagers, and adults with amblyopia: a randomized clinical trial. IAMA Ophthalmol. 2018;136:172-81.

28. Žiak P. Holm A Halička J et al. Amblyopia treatment of adults with dichoptic training using the virtual reality oculus rift head-mounted display: preliminary results. BMC Ophthalmol. 2017;17:105.

29. KnoX PJ, Simmers AJ, Gray LS, Cleary M. An exploratory study: prolonged periods of binocular stimulation can provide an effective treatment for childhood amblyopia. Invest Ophthalmo Vis Sci. 2012;53:817-24.

30. Bossi M, Tailor VK, Anderson EJ, et al. Binocular therapy for childhood amblyopia improves vision without breaking interocular suppression. Invest Ophthalmol Vis SCi. 2017;58:3031-43. 